\title{
A PRELIMINARY REPORT OF SOME EXPERIMENTS UPON THE CHEMISTRY OF THE DIPHTHERIA ANTITOXIN.
}

\author{
By T. G. Bronie, M.D., Lecturer on Physiology, St. Thomas's Hospital \\ Medical Sehool.
}

From the Conjoint Laboratories of the Royal Colleges of Physicians (Lond.)
and Surgeons (Eng.)

THE experiments of which this short paper gives a preliminary account, were initially undertaken with the view of determining the nature of the substance or substances present in antitoxic diphtheria serum, which cause the production of a rash, and, if possible, to discover some simple process by which it might be removed without injury to the antitoxin.

That the production of a rash is not due to the antitoxin itself, is clearly pointed to by the fact that the power of rash production of a serum in no way corresponds to its antitoxic strength. It does, however, apparently depend upon the existing condition of the horse from which it has been derived, the same horse tending to give a serum of marked rash-producing properties.

In the first experiments extractions were made with various liquids; ether, absolute alcohol, acetone, and chloroform being employed to find out whether any substance producing a rash could be obtained in the extract. The method employed was to dry the serum by pouring it in a thin film over a glass plate, the temperature of which was kept at about $40^{\circ} \mathrm{C}$. In this way the solid parts were obtained in the form of bright amber-coloured plates, which were readily broken into a very fine powder in a mortar. The powder redissolved in water fairly easily, but not quite completely, giving a solution which was only distinguished from the original serum by a slight degree of opalescence. The fine powder was then extracted with the different solvents, after which it was thoroughly dried at a low temperature, redissolved in distilled water, and its strength of antitoxin tested. In the earlier experiments acetone and ether were used. Thus, in one experiment, the dried powder from 30 c.c: of serum was placed under acetone, which was frequently renewed, for three days, after which it was extracted with acetone containing oxalic acid in solution (a 1 per 
cent. solution) four successive times, the whole process lasting threequarters of an hour. It was then thoroughly washed for a further half-hour with a large bulk of acetone, in order to remove any oxalic acid, and finally dried until all the acetone had been driven off. The dried powder was then dissolved in a little distilled water, so as to bring the solution to the same bulk as that of the original serum taken. Most of the powder dissolved very readily, but a few rather coarse flocculi were left undissolved. In order to take these up, a crystal of sodium chloride was added, which, however, did not completely dissolve them. The solution was consequently filtered, and as thus obtained had a pale yellow colour, much lower in tint than the original serum, and was slightly opalescent. It gave no precipitate with calcium chloride, and was therefore free from oxalic acid. It was then tested on guinea-pigs, to determine its strength. It was found in this case that the prolonged extraction with acetone had destroyed some of the antitoxin, for it only showed a little more than two-thirds of its initial strength. The acetone extract was next evaporated to dryness at a low temperature, under diminished pressure, and was found to leave a small quantity of a gummy residue, which had a very irritating and pungent odour, especially when warm. A little of the pungent vapour, accidentally acting on the conjunctiva, produced very painful smarting, which persisted for some time. ${ }^{1}$ The extraction with acetone containing oxalic acid in solution was carried out, because it was thought that there might be some organic acid or conjugated acid present, which might be the cause of the rash.

A similar experiment was also made where, however, ether was used as the extracting agent, and care was taken that the extraction should occupy as short a time as possible. The extraction with ether containing an acid in solution was in this case omitted. An almost identical result was obtained, for some of the antitoxic properties were found to have been destroyed. The ethereal extract again yielded the same irritating substance, which had been dissolved out by acetone. This substance, derived from the two sources, was dissolved in a little ether and injected into a white rabbit and a white guineapig, to see if any rash would be produced by it in either case. No result of any kind was obtained. A negative result is, however, in no way decisive, for it is extremely difficult, if not impossible, to produce a rash in these animals. The only effect which has been produced is shown in a falling off of the hair.

The experiments so far had indicated that the procedure adopted had led to the destruction of some of the antitoxin. It was therefore necessary to determine whether this was due to the drying or to the

I It is very important that the acetone should be quite pure. Ordinary "pure "acetone is usually acid to litmus, and leaves a brownish syrupy residue on evaporation. The acetone used in these experiments was freshly distilled, a little lime being added to it in the distilling flask, to neutralise any acid which might be present. 
direct action of the extractive. A small quantity of serum was therefore dried by the method previously adopted, and when thoroughly dry it was redissolved and its strength tested. As in the previous case the solution obtained was not complete, and some opalescence was present. The solution was found to have lost some of its strength, though not so much as in the first two experiments described. This is most probably due, to a certain extent, to the greater degree of dehydration obtaining in those cases, for acetone, especially, tends to remove all the water still present in the powder after drying in the air. This is further confirmed by later experiments in other directions.

It soon became evident that the only satisfactory method which could be adopted was, in the first place, to find out more of the reactions of antitoxin, in order to gain a knowledge of the reagents which might with safety be employed in its purification. Drying, if carried out thoroughly, is a process which, as we have seen, it may be necessary to avoid. ${ }^{1}$

If acetone be added to serum in sufficient bulk, all the proteids are completely precipitated. I then proceeded to examine in which part the antitoxin was to be found. If about 15 c.c. of acetone be added to each 10 c.c. of serum, the precipitate is coarsely flocculent, soon settles down, and will rapidly filter. The filtrate was taken, and the acetone quickly evaporated off by distilling it at about $30^{\circ} \mathrm{C}$. under diminished atmospheric pressure. The aqueous solution remaining was then tested and found to contain no antitoxin. The white precipitate while still moist was dissolved in a 1 per cent. $\mathrm{NaCl}$ solution, in which it rapidly and completely dissolved, giving an almost colourless opalescent solution, which was then freed from the remaining traces of acetone. The antitoxin was found in this solution, and to the same amount as originally present in the serum. It was hoped that by this method the ash-producing substance would be left in the filtrate, and that the solution of the precipitate would be free from them. A considerable bulk of serum was therefore treated in this manner, but the results of the treatment of patients with this serum are not yet known. In connection with this precipitation of the antitoxin with acetone, an important point is with regard to the length of time the acetone is allowed to remain in contact with the precipitate. If rapidly filtered off and redissolved, without being allowed to dry, solution occurs rapidly and completely, and no antitoxin is lost. In another experiment, however, a small quantity was kept under the acetone for 24 hours. It was then found that re-solution was much more difficult and imperfect, and that much

${ }^{1}$ In a recent paper Behring (Fortschr. d. Med., Berlin, 1897, Bd. xv. p. 1) recommends that the serum should be dried. Dried serum he finds does not lose any of its strength on keeping, as so frequently happens with the strong serums preserved in the ordinary way. Behring does not, however, directly state that antitoxin is not destroyed in the drying process, nor again to what extent the drying is carried. 
of the antitoxin had been destroyed. An almost identical result was obtained by employing absolute alcohol instead of acetone, though here the destruction of the antitoxin occurs much more rapidly.

One of the disadvantages of working by this method is, that all the proteids are carried down with the antitoxin, and as these are present in very large quantities, it is not possible to obtain any degree of concentration.

It is known that the antitoxins are precipitated by saturation of their solutions with many of the neutral salts. I have confirmed this for the diphtheria antitoxin for magnesium sulphate and half saturation with ammonium sulphate. As a general result of all experiments in this and other directions, I have found that diphtheria antitoxin is completely precipitated from a solution by any means which removes the globulins.

A knowledge of this point suggests one or two queries. In the first place, is the antitoxin a proteid? In that case it belongs to the group of globulins. Or, on the other hand, is the antitoxin simply carried down by the first voluminous precipitate caused to appear in a solution containing it? I have attempted to gain an answer to these points in several ways. Some of the precipitate obtained by adding acetone to diphtheria serum was thoroughly extracted with a saturated solution of ammonium sulphate, containing a trace of ammonia. After a time it was filtered, and the filtrate was found to yield a faint xanthoproteic reaction. No antitoxin could, however, be detected in it. A similar result was obtained when a halfsaturated solution was employed instead of a saturated.

In the next instance the method of fractional precipitation was employed. The globulins were precipitated in four parts by the gradual addition of ammonium sulphate until half-saturation was reached. It was found, however, that each of the four successive precipitations contained antitoxin, and moreover in about equal bulks, so that the method did not offer any promise of a possibility of obtaining a stronger solution than could be obtained by redissolving the precipitated globulins as a whole. The fact that each of the four fractions contained approximately equal quantities of antitoxin tends also to prove that the precipitation of the globulins does not carry down with it the antitoxin by a simple mechanical process. This was further emphasised by the following experiment:-To 5 c.c. of serum 1 c.c. of a strong sodium silicate solution was added, thoroughly mixed, and then acetic acid added drop by drop until the whole had set to a solid mass, by the production of a big gelatinous precipitate of silicic acid. This was then thoroughly broken up and filtered. Both filtrate and precipitate were then examined for antitoxin, which was found in both.

The action of acids and alkalies was also examined. It was found that quite small quantities of caustic potash very rapidly 
destroyed the antitoxin. The action of dilute sodium carbonate was found to be much slower, though here again some destruction occurred. Of the acids tested in this respect I have only employed organic acids in dilute solution. Thus, in one experiment, 1.5 c.c. of a 1 per cent. solution of oxalic acid was added to 5 e.c. of serum, and the mixture kept at $36^{\circ} \mathrm{C}$. for 48 hours. A considerable amount of antitoxin was still found in the solution after this treatment.

These experiments were arranged as preliminary to the determination as to whether the antitoxin would be destroyed by the action of the digestive ferments, pepsin and trypsin. These latter are not, however, in a sufficiently complete state to enable me to record them. They were carried out with a view to showing, that if they were not destroyed by the ferment, they were probably not of the nature of an ordinary globulin. If, however, they are destroyed by the ferment, the fact would tend to show that they are proteid in nature.

In a recent paper, C. J. Martin ${ }^{1}$ has shown that crystalloids may be rapidly separated from colloids by filtration, under high pressure, through a film of gelatine, through the interstices of which the small molecules of the crystalloid can pass, while the larger molecules of the colloid are kept back. I have applied the method he uses to diphtheria serum, and find that the filtrate obtained is completely free from antitoxin, a fact which further confirms the view to which many of my experiments tend, that the antitoxin is a proteid, and that, as shown by its solubilities, it must belong to the globulin class.

The knowledge that antitoxin will not pass through gelatine will prove of value in several directions. By precipitating the globulins, and with them the antitoxins, we can immediately remove about one-half of the total proteids, and with them in all probability the substance producing the rash. A previous objection to the use of neutral salts was the difficulty of their complete removal from the precipitate; this can however, by this method, be readily attained in a fairly rapid manner, whereas, previously, it became necessary to dialyse away the salts. Moreover, by the same method, it is possible in a convenient way to concentrate a solution of the antitoxin to any required degree, and by a redilution and reconcentration we may completely remove from the serum all of those soluble substances which can be made to pass through a gelatine film.

In conclusion, I wish to express my thanks to Dr. Sims Woodhead, for his kindness and criticism during the course of these experiments, and for carrying out for me most of the actual injections.

These experiments have been carried out by the aid of a grant from the Goldsmith's Company.

${ }^{1} J o u m$. Physiol., Cambridge and London, 1896, vol. xx. p. 364. 